\title{
DISTRIBUIÇÃO, PROBABILIDADE DE OCORRÊNCIA E PERÍODO DE RETORNO DOS ÍNDICES DE EROSIVIDADE EI 30 E KE>25 EM SEROPÉDICA - RJ
}

\section{DANIEL F. DE CARVALHO ${ }^{1}$, RORIZ L. MACHADO ${ }^{2}$, ADÃO W. P. EVANGELISTA ${ }^{3}$, JOSEPH K. KHOURY JÚNIOR ${ }^{4}$, LEONARDO D. B. DA SILVA ${ }^{5}$}

RESUMO: o presente trabalho foi desenvolvido com o objetivo de estudar as características da erosividade da chuva em Seropédica (RJ), quanto à sua distribuição, probabilidade de ocorrência e período de retorno. Para isso, foi utilizada uma série mensal de dados pluviométricos referente ao período de 1973 a 2002 e, com o auxílio de modelos ajustados para a região, foi possível obter os índices mensais e anuais de erosividade $\mathrm{EI}_{30}$ e $\mathrm{KE}>25$. Com base nos resultados obtidos, foi possível concluir que: a) os valores médios anuais de $E_{30}$ e de $K E>25$ foram de $5.960,4 \mathrm{MJ} \mathrm{mm} \mathrm{ha}^{-1} \mathrm{~h}^{-1}$ e de 99,2 $\mathrm{MJ} \mathrm{ha}^{-1}$, respectivamente, e estão associados a períodos de retorno de 1,97 ano, com uma probabilidade de ocorrência de $50,82 \%$; e b) valores anuais de $\mathrm{EI}_{30}$ da ordem de $5.995 ; 7.262 ; 7.684 ; 7.895 ; 8.022$ e $8.064 \mathrm{MJ} \mathrm{mm} \mathrm{ha}^{-1} \mathrm{~h}^{-1}$ e de KE>25 da ordem de 99,8; 122,7; 130,$3 ; 134,1 ; 136,4$ e $137,1 \mathrm{MJ}^{-1}$, são esperados, em média, uma vez a cada $2 ; 5 ; 10 ; 20 ; 50$ e 100 anos, respectivamente.

PALAVRAS-CHAVE: erosividade da chuva, erosão hídrica, função de probabilidade.

\section{EROSIVITY INDEXES EI I0 $_{30}$ AND KE >25 AT SEROPEDICA, RIO DE JANEIRO STATE, BRAZIL: DISTRIBUTION, OCCURRENCE PROBABILITY AND RETURN PERIOD}

\begin{abstract}
This work was carried out in order to study the distribution, the occurrence probability and the return period of the rainfall erosivity in Seropédica city, Rio de Janeiro State, Brazil. It was considered a continuous rain gauges series for the period from 1973 to 2002, and by using specific adjustment models for the region, it was determined the annual and monthly erosivity indexes $\mathrm{EI}_{30}$ and $\mathrm{KE}>25$. With the obtained results, it was possible to conclude that: a) the annual erosivity indexes values $\mathrm{EI}_{30}$ and $\mathrm{KE}>25$ were 5,960.4 $\mathrm{MJ} \mathrm{mm} \mathrm{ha}{ }^{-1} \mathrm{~h}^{-1}$ and $99.2 \mathrm{MJ} \mathrm{ha}^{-1}$, respectively, which was expected to occur at least once every 1.97 year, with an occurrence probability of $50.82 \%$; and b) annual values $\mathrm{EI}_{30}$ of 5,$995 ; 7,262 ; 7,684 ; 7,8958,022$ and $8,064 \mathrm{MJ} \mathrm{mm} \mathrm{ha}{ }^{-1} \mathrm{~h}^{-1}$ and annual values $\mathrm{KE}>25$ of $99.8 ; 122.7 ; 130.3 ; 134.1 ; 136.4$ and 137.1 $\mathrm{MJ} \mathrm{ha}^{-1}$ are expected at least once every $2 ; 5 ; 10 ; 20 ; 50$ and 100 years, respectively.
\end{abstract}

KEYWORDS: rainfall erosivity, soil erosion, probability function.

\section{INTRODUÇÃO}

A erosividade é um índice numérico que expressa a capacidade da chuva, esperada em uma localidade, de causar erosão em uma área sem proteção (FAO, 1967). Esse fator tem sido considerado um dos mais importantes componentes na estimativa da erosão hídrica ao se utilizarem métodos indiretos, como a Equação Universal de Perda de solo (WISCHMEIER \& SMITH, 1958), pois permite quantificar o efeito do impacto das gotas de chuvas sobre o solo, possibilitando a estimativa da sua erodibilidade. De acordo com VAL et al. (1986), a variação deste índice ao longo do ano define a melhor época para o estabelecimento das práticas de manejo e conservação do solo, além de servir como parâmetro de suporte a trabalhos de extensão e assistência rural (BERTOL, 1994), pois permite definir épocas críticas quanto à ocorrência da erosão. A situação torna-se mais

\footnotetext{
${ }^{1}$ Prof. Associado, Departamento de Engenharia, UFRRJ, Seropédica - RJ, carvalho@ufrrj.br. Bolsista do CNPq

${ }^{2}$ Prof. do IF Goiano, BR 153, km 633, Caixa Postal 92, Morrinhos - GO, rorizmachado@ifgoiano.edu.br

${ }^{3}$ Prof. Adjunto, Setor de Engenharia Rural, UFG, Goiânia - GO, awpego@ bol.com.br

${ }^{4}$ Prof. Adjunto, Departamento de Engenharia de Produção e Mecânica, UFV, Viçosa - MG, kalil@ufv.br

${ }^{5}$ Prof. Adjunto, Departamento de Engenharia, UFRRJ, Seropédica - RJ, irriga@ufrrj.br

Recebido pelo Conselho Editorial em: 25-6-2008

Aprovado pelo Conselho Editorial em: 13-1-2010
} 
dramática no momento em que o aumento de utilização agrícola das terras vem, há alguns anos, comprometendo o uso dos solos, muitos dos quais já não atendem à necessidade de produção autossustentável em virtude da aceleração das taxas de erosão (DIAS \& SILVA, 2003).

A erosividade é exclusivamente função das características físicas da chuva, como quantidade, intensidade, diâmetro de gotas, velocidade terminal e energia cinética (HUDSON, 1973); e dentre os índices existentes, destaca-se o $\mathrm{EI}_{30}$, definido pelo produto entre a energia cinética da chuva e sua intensidade máxima em $30 \mathrm{~min}$. De acordo com WISCHMEIER \& SMITH (1958), o EI 30 representa os efeitos do impacto das gotas e da turbulência, combinados com a capacidade de transporte do escoamento superficial, e tem sido utilizado em diversos países, inclusive no Brasil. Entretanto, sua utilização passou a ser questionada em países tropicais e subtropicais, que se caracterizam por apresentar chuvas com intensidades de até $150 \mathrm{~mm} \mathrm{~h}^{-1}$, sendo propostos, em razão disso, índices alternativos como o KE>25 (HUDSON, 1973), que considera o cálculo da energia cinética da chuva apenas para intensidades de precipitação maiores do que $25 \mathrm{~mm} \mathrm{~h}^{-1}$. De acordo com LAL (1976), o índice KE>25 é um índice de mais fácil obtenção, uma vez que dispensa o uso da intensidade máxima em trinta minutos.

Estimativas anuais do índice de erosividade $\mathrm{EI}_{30}$, expresso em $\mathrm{MJ} \mathrm{mm} \mathrm{ha}^{-1} \mathrm{~h}^{-1}$ ano ${ }^{-1}$, tem sido realizadas para diferentes regiões do País. Dentre eles, podem ser citados os trabalhos de BERTONI \& LOMBARDI NETO (1990), no litoral do Estado de São Paulo (6.000 a 7.500); RUFINO et al. (1993), no Estado do Paraná, (5.500 a 12.000); MAIA NETO \& CHAVES (1997), no Estado da Paraíba (2.000 a 8.000) e GONÇALVES (2002) e MONTEBELLER (2005), no Estado do Rio de Janeiro (3.440 a 14.800). Para o índice KE>25, MONTEBELLER (2005) encontrou uma variação de 46,58 a $166,70 \mathrm{MJ}^{-1}$.

Além da magnitude do evento, é fundamental a realização de estudos que levem em consideração a sua frequência e suas probabilidades teóricas de ocorrência. Assim, as informações estimadas ou coletadas nos postos hidrométricos podem ser analisadas estatisticamente, verificando-se com que frequência elas assumiram cada magnitude, sendo de grande importância para um adequado planejamento de práticas conservacionistas e de projetos de obras hidráulicas em geral (VILLELA \& MATOS, 1975).

Diante do exposto, desenvolveu-se este trabalho com o objetivo de estudar as características da erosividade da chuva em Seropédica - RJ, quanto à sua distribuição, probabilidade de ocorrência e período de retorno.

\section{MATERIAL E MÉTODOS}

O trabalho foi desenvolvido com dados de chuva coletados na estação denominada Ecologia Agrícola e registrada no INMET com o código 83741. Localizada no município de Seropédica - RJ, a referida estação encontra-se nas coordenadas $22^{\circ} 48^{\prime}$ de latitude sul e $43^{\circ} 41^{\prime}$ de longitude oeste e apresenta altitude de aproximadamente $33 \mathrm{~m}$. Segundo a classificação de Köeppen, o clima predominante na região é o Aw, com chuvas concentradas no período de novembro a março, precipitação média anual de $1.213 \mathrm{~mm}$ e temperatura média anual de $24,5{ }^{\circ} \mathrm{C}$ (CRUZ, 2005).

Foram utilizados dados diários de precipitação coletados a partir de 1940, com os quais foram obtidas as precipitações mensais e, posteriormente, as precipitações totais anuais. Com o objetivo de avaliar a adequação do tamanho da série pluviométrica a ser utilizada para a estimativa dos índices de erosividade $\mathrm{EI}_{30}$ e $\mathrm{KE}>25$, foi avaliado, previamente, o maior período de anos possível em que a lâmina precipitada obtida da série pluviométrica fosse semelhante estatisticamente à lâmina pluviométrica referente à série pluviográfica empregada na obtenção das eqs.(1) e (2). Para isso, foi utilizada a análise de Intervalos de Confiança, conforme MORETI et al. (2003a).

Após a definição da série de dados e com base nos totais médios mensais de precipitação, os índices de erosividade $\mathrm{EI}_{30}$ foram obtidos a partir da equação proposta por CARVALHO et al. (2005): 


$$
\mathrm{EI}_{30}=7,171 \mathrm{P}+211,82
$$

em que,

$\mathrm{P}$ - precipitação média mensal, em mm.

Para a obtenção dos índices KE>25 médios mensais, foi utilizada a eq.(2), proposta por MONTEBELLER (2005):

$$
\mathrm{KE}>25=0,5212 \mathrm{Rc}+1,75533
$$

em que,

Rc-coeficiente de chuva, obtido pelo quociente entre o quadrado da precipitação pluviométrica média mensal e a precipitação pluviométrica média anual.

Os índices de erosividade, mês a mês, dentro de cada ano, foram então estimados conforme MORETI et al. (2003a), a partir do quadro geral de distribuição da precipitação pluviométrica para o município de Seropédica, por meio de regra de três simples, da seguinte forma:

$$
\mathrm{Ei}=\frac{(\mathrm{P} I E)}{\mathrm{Pa}}
$$

em que,

Ei - índice de erosividade de um mês i contido num ano j da série de dados;

$P$ - total precipitado num mês i contido num ano $\mathrm{j}$ da série de dados;

IE - índice de erosividade ( $\mathrm{EI}_{30}$ ou $\left.\mathrm{KE}>25\right)$ médio mensal, no qual o mês i está contido na série de dados, e

$\mathrm{Pa}$ - precipitação média mensal no qual o mês i está contido na série de dados.

A obtenção dos índices de erosividade mês a mês, dentro de cada ano, permitiu a obtenção do quadro final de índices de erosividade, $\mathrm{EI}_{30}$ e KE>25 para Seropédica, no período de 1973 a 2002. A partir daí, o somatório dos índices de erosividade mês a mês, dentro de cada ano, constituiu o índice de erosividade para determinado ano.

O período de retorno $(\mathrm{T})$ e a probabilidade de ocorrência (Pr) foram obtidos conforme VILLELA \& MATOS (1975), utilizando as eqs. (4) e (5), respectivamente, a seguir descritas:

$$
\mathrm{T}=\frac{\mathrm{N}+1}{\mathrm{~m}}
$$

em que,

$\mathrm{N}$ - número de anos da extensão da série parcial de dados, e

m - número de ordem do índice de erosividade.

$$
\operatorname{Pr}=\frac{100}{\mathrm{~T}}
$$

Nas eqs.(4) e (5), T é expresso em anos e Pr em \%.

Com auxílio de uma planilha eletrônica, os valores dos índices de erosividade foram ordenados em ordem decrescente e associados a um valor de ordem que variou de 1 a 30 . Em virtude de trabalhos anteriores terem sido desenvolvidos utilizando a função Log-normal (MORETI et al., 2003a; EVANGELISTA et al., 2006), típica de eventos hidrológicos (ROQUE et al., 2001), foi realizado inicialmente um ajuste para esta distribuição. Para isso, foi estimado o valor do coeficiente de frequência, utilizando a eq.(6):

$$
\mathrm{z}=\frac{\log (\mathrm{IE})-\overline{\mathrm{IE}} \mathrm{l}}{\mathrm{DPl}}
$$

em que, 
IE - índice de erosividade;

$\overline{\mathrm{IE}}$ - média do logaritmo dos valores de IE, e

DP1 - desvio-padrão do logaritmo dos valores de IE.

Em seguida, foram estimados os valores teóricos de probabilidade (Prt) por meio da função distribuição de probabilidade Log-normal, utilizando-se do pacote de ferramentas do programa computacional MATLAB (MathWorks, Inc., 2000):

$$
\operatorname{Prt}=\frac{1}{\mathrm{IE} \mathrm{DP} \sqrt{2 \pi}} \mathrm{E}^{\frac{-(\ln (\mathrm{IE})-\overline{\mathrm{I}})^{2}}{2 \mathrm{DP}^{2}}}
$$

em que,

$\overline{\mathrm{IE}}$ - média dos valores de IE, e

DP - desvio-padrão dos valores de IE.

A fim de verificar o ajuste dos dados a essa distribuição, foi utilizado o teste de aderência de Kolmogorov-Smirnov (K-S), a 5\% de probabilidade (EVANGELISTA et al., 2006). Assim, para cada valor de ordem, foi estimada a diferença entre o valor de probabilidade calculado pela eq.(5) e o valor teórico obtido pela eq.(7). As máximas diferenças obtidas para os índices $\mathrm{EI}_{30}$ e $\mathrm{KE}>25$ foram de 0,10 e 0,12 , respectivamente, inferiores ao valor tabelado $(0,24)$ para o teste K-S, para o nível de significância utilizado. Portanto, foi possível constatar que os conjuntos de dados estimados de $\mathrm{EI}_{30}$ e $\mathrm{KE}>25$ poderiam razoavelmente ser considerados como provenientes de uma população com aquela distribuição de probabilidade. Finalmente, com base nos valores teóricos de probabilidade, do período de retorno e dos índices de erosividade, foram confeccionadas, no ambiente MATLAB (2000), as curvas e equações de ajuste linear, possibilitando a estimativa dos índices $\mathrm{EI}_{30}$ e KE>25 para diferentes valores de probabilidade de ocorrência.

\section{RESULTADOS E DISCUSSÃO}

Na Figura 1, apresenta-se a análise de Intervalos de Confiança das lâminas precipitadas provenientes da série pluviométrica e referentes à série pluviográfica empregada na obtenção dos modelos utilizados neste trabalho [eqs.(1) e (2)]. Com base nessa análise, foi escolhido o intervalo de anos de 1973 a 2002 da série pluviométrica disponível, o qual apresentou, para todos os meses, médias de precipitação próximas às medias de precipitação registradas na estação pluviográfica, ou que, pelo menos, os intervalos de confiança de ambas se sobrepuseram como observado nos meses de março e agosto.

Na Tabela 1, estão apresentados os valores médios mensais de chuva, coeficiente de chuva e índices de erosividade adotados neste trabalho, para o município de Seropédica, no período de 1973 a 2002. Pela Tabela 1, percebe-se que a precipitação média anual em Seropédica é de 1.185,6 mm e que os índices de erosividade médios anuais obtidos foram de 5.960,4 $\mathrm{MJ} \mathrm{mm} \mathrm{ha}^{-1} \mathrm{~h}^{-1}\left(\mathrm{EI}_{30}\right) \mathrm{e}$ 99,2 $\mathrm{MJ} \mathrm{ha}^{-1}$ (KE>25), com desvios-padrão de $377,8 \mathrm{MJ} \mathrm{mm} \mathrm{ha}^{-1} \mathrm{~h}^{-1}$ e $5,1 \mathrm{MJ} \mathrm{ha}^{-1}$, respectivamente.

Os valores mensais de $\mathrm{EI}_{30}$ variaram de 14,9 $\mathrm{MJ} \mathrm{mm} \mathrm{ha}^{-1} \mathrm{~h}^{-1}$, no mês de julho, a 1.090,2 $\mathrm{MJ} \mathrm{mm} \mathrm{ha}{ }^{-1} \mathrm{~h}^{-1}$, no mês de dezembro. Ao analisar todos os dados da série, foi verificado que o mesmo índice variou de 0,0 MJ mm ha ${ }^{-1} \mathrm{~h}^{-1}$, em julho de 1974 e fevereiro de 1984, a 2.512 $\mathrm{MJ} \mathrm{mm} \mathrm{ha}^{-1} \mathrm{~h}^{-1}$, correspondendo ao mês de fevereiro de 1994. Quanto ao KE>25, valor zero foi observado para o período de junho a agosto para diferentes anos, sendo o maior valor encontrado de 75,0 $\mathrm{MJ} \mathrm{ha}^{-1}$, em fevereiro de 1988. 


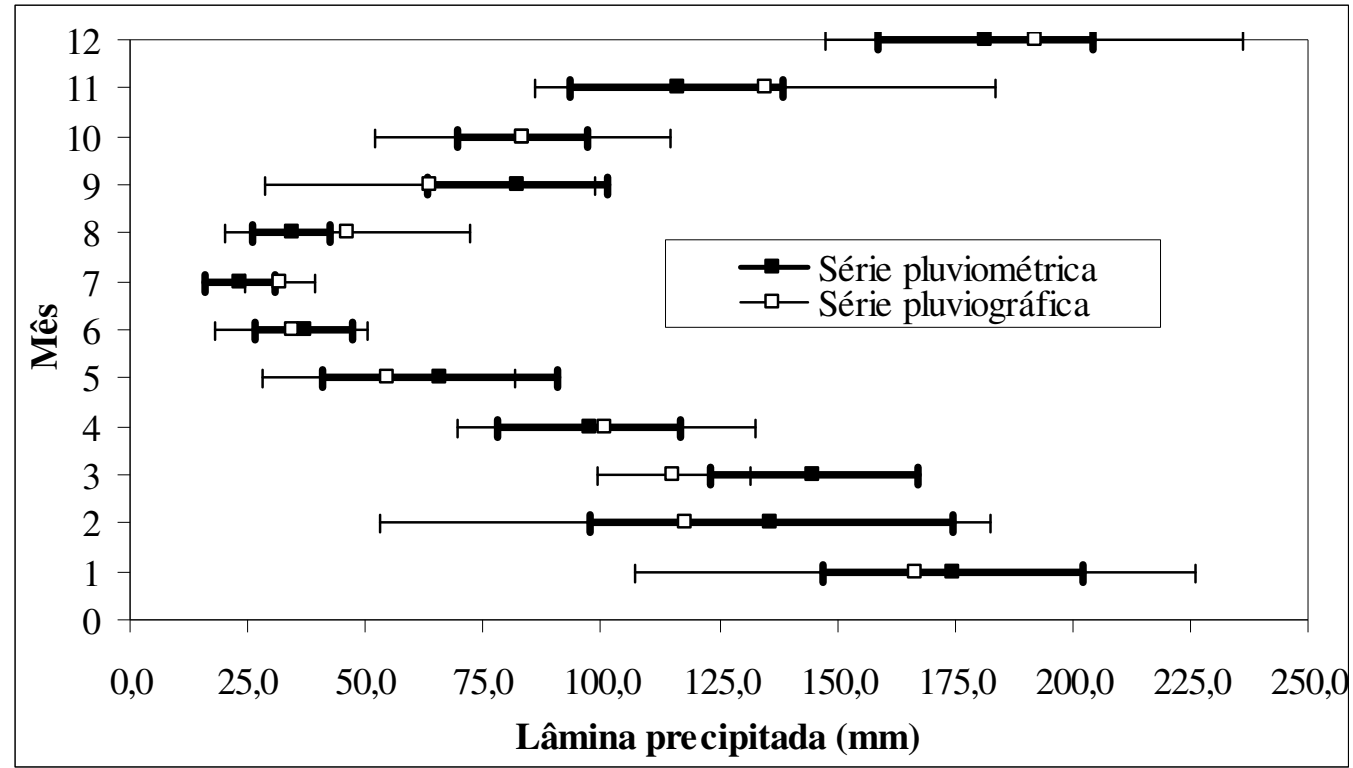

FIGURA 1. Médias e intervalo de confiança da precipitação anual obtidos entre séries pluviométrica e pluviográfica em Seropédica - RJ. Averages and confidence interval of the annual rainfall obtained from pluviometric and pluviographic series in Seropédica, Rio de Janeiro State, Brazil.

TABELA 1. Valores médios mensais de chuva, coeficiente de chuva e índices de erosividade para Seropédica - RJ, avaliados no período de 1973 a 2002. Medium monthly values of rain, rain coefficient and erosivity index for Seropédica (Rio de Janeiro State, Brazil), evaluated from 1973 to 2002.

\begin{tabular}{|c|c|c|c|c|}
\hline \multirow{2}{*}{ Mês } & Chuva & $\mathrm{Rc}$ & $\mathrm{EI}_{30}$ & $\mathrm{KE}>25$ \\
\hline & \multicolumn{2}{|c|}{$\mathrm{mm}$} & MJ mm ha ${ }^{-1} h^{-1}$ mês $^{-1}$ & $\mathrm{MJ} \mathrm{ha}^{-1}$ \\
\hline janeiro & 174,8 & 29,6 & $1.041,8$ & 17,2 \\
\hline fevereiro & 136,0 & 22,0 & 763,8 & 13,2 \\
\hline marco & 145,1 & 19,7 & 828,9 & 12,0 \\
\hline abril & 97,5 & 10,5 & 487,5 & 7,2 \\
\hline maio & 65,9 & 4,6 & 260,7 & 4,2 \\
\hline junho & 37,0 & 1,8 & 53,6 & 2,7 \\
\hline julho & 31,6 & 1,2 & 14,9 & 2,4 \\
\hline agosto & 34,4 & 1,4 & 34,6 & 2,5 \\
\hline setembro & 82,2 & 7,3 & 377,7 & 5,5 \\
\hline outubro & 83,4 & 7,0 & 386,5 & 5,4 \\
\hline novembro & 116,0 & 13,9 & 620,3 & 9,0 \\
\hline dezembro & 181,6 & 30,8 & $1.090,2$ & 17,8 \\
\hline Total & $1.185,6$ & ---- & $5.960,4$ & 99,2 \\
\hline Desvio-padrão & ---- & ---- & 377,8 & 5,1 \\
\hline
\end{tabular}

Na Tabela 2, são apresentados os valores da probabilidade de ocorrência e o período de retorno dos índices anuais de erosividade $\left(\mathrm{EI}_{30}\right.$ e $\left.\mathrm{KE}>25\right)$, para o município de Seropédica, no período de 1973 a 2002 . Nesta Tabela, é possível perceber que os valores anuais de $\mathrm{EI}_{30}$ variaram de 3.350,6 a 9.792,9 $\mathrm{MJ} \mathrm{mm} \mathrm{h}^{-1} \mathrm{ha}^{-1}$ e corresponderam aos anos de 1984 e 1996, respectivamente.

Para o índice KE $>25$, nesses mesmos anos, os valores estimados foram de 56,8 e 158,5 $\mathrm{MJ} \mathrm{ha}^{-1}$. Analisando a série de dados pluviométricos, foi possível constatar que os anos de 1984 e 1996 foram, respectivamente, aqueles com menor $(671,8 \mathrm{~mm})$ e maior $(1.876,4 \mathrm{~mm})$ magnitudes pluviométricas, influenciando, de maneira expressiva, nos índices de erosividade. 
Quanto a $\mathrm{T}$ e $\mathrm{Pr}$, os maiores valores de $\mathrm{EI}_{30}$ e $\mathrm{KE}>25$ apresentados na Tabela 2 estão associados, respectivamente, a 31 anos e 3,2\%, enquanto o período de retorno de 1,0 ano e a probabilidade de 96,8\% estão associados, respectivamente, a 3.350,6 $\mathrm{MJ} \mathrm{mm} \mathrm{h}^{-1} \mathrm{ha}^{-1} \mathrm{ano}^{-1} \mathrm{e}$ $56,8 \mathrm{MJ} \mathrm{ha}^{-1}$ ano $^{-1}$.

TABELA 2. Índice pluviométrico anual, período de retorno $(\mathrm{T})$ e probabilidade de ocorrência $(\mathrm{Pr})$ dos índices anuais $\mathrm{EI}_{30}$ e $\mathrm{KE}>25$ para Seropédica $(\mathrm{RJ})$, com base nas chuvas do período de 1973 a 2002. Annual rainfall index, return period (T) and occurrence probability (Pr) of the annual indexes $\mathrm{EI}_{30}$ and $\mathrm{KE}>25$ for Seropédica (Rio de Janeiro State, Brazil), based on the rainfall from 1973 to 2002.

\begin{tabular}{|c|c|c|c|c|c|c|c|c|}
\hline \multicolumn{2}{|c|}{ Chuva } & \multicolumn{2}{|c|}{$\mathrm{EI}_{30}$} & \multicolumn{2}{|c|}{ KE $>25$} & \multirow{2}{*}{$\begin{array}{l}\text { Número de } \\
\text { Ordem }\end{array}$} & \multirow{2}{*}{$\begin{array}{c}\mathrm{T} \\
\text { (anos) }\end{array}$} & \multirow{2}{*}{$\begin{array}{l}\mathrm{Pr} \\
(\%)\end{array}$} \\
\hline Ano & Valor & Ano & Valor & Ano & Valor & & & \\
\hline 1996 & $1.876,4$ & 1996 & $9.792,9$ & 1996 & 158,5 & 1 & 31,00 & 3,2 \\
\hline 1985 & $1.485,5$ & 1985 & $7.906,8$ & 1988 & 136,1 & 2 & 15,50 & 6,5 \\
\hline 1988 & $1.415,4$ & 1988 & $7.274,6$ & 1992 & 134,2 & 3 & 10,33 & 9,7 \\
\hline 1983 & $1.413,1$ & 1980 & $7.041,6$ & 1985 & 129,9 & 4 & 7,75 & 12,9 \\
\hline 1980 & $1.383,8$ & 1991 & $7.033,6$ & 1983 & 117,1 & 5 & 6,20 & 16,1 \\
\hline 1992 & $1.371,6$ & 1992 & $6.837,4$ & 1991 & 116,3 & 6 & 5,17 & 19,4 \\
\hline 1995 & $1.362,2$ & 1973 & $6.681,5$ & 1993 & 108,9 & 7 & 4,43 & 22,6 \\
\hline 1991 & $1.347,1$ & 1998 & $6.676,6$ & 1997 & 108,5 & 8 & 3,88 & 25,8 \\
\hline 1998 & $1.318,6$ & 1993 & $6.654,6$ & 1977 & 107,4 & 9 & 3,44 & 29,0 \\
\hline 1973 & $1.312,8$ & 1983 & $6.649,4$ & 1986 & 104,1 & 10 & 3,10 & 32,3 \\
\hline 1976 & $1.286,4$ & 1995 & $6.640,7$ & 1975 & 103,9 & 11 & 2,82 & 35,5 \\
\hline 1993 & $1.285,4$ & 1997 & $6.276,2$ & 1980 & 103,1 & 12 & 2,58 & 38,7 \\
\hline 1987 & $1.218,1$ & 1975 & $6.245,9$ & 1998 & 100,4 & 13 & 2,38 & 41,9 \\
\hline 1977 & $1.210,5$ & 1987 & $6.173,0$ & 1974 & 100,1 & 14 & 2,21 & 45,2 \\
\hline 1986 & $1.188,0$ & 1977 & $6.086,8$ & 1982 & 99,8 & 15 & 2,07 & 48,4 \\
\hline 1997 & $1.186,1$ & 1976 & $6.029,7$ & 1973 & 99,3 & 16 & 1,94 & 51,6 \\
\hline 1975 & $1.173,5$ & 1982 & $6.009,8$ & 1976 & 97,8 & 17 & 1,82 & 54,8 \\
\hline 1982 & $1.167,3$ & 1986 & $5.716,6$ & 2000 & 97,6 & 18 & 1,72 & 58,1 \\
\hline 1994 & $1.114,5$ & 1994 & $5.451,9$ & 1995 & 97,0 & 19 & 1,63 & 61,3 \\
\hline 1979 & $1.104,2$ & 2000 & $5.353,5$ & 1994 & 94,9 & 20 & 1,55 & 64,5 \\
\hline 2000 & $1.098,4$ & 1979 & $5.271,6$ & 1987 & 92,2 & 21 & 1,48 & 67,7 \\
\hline 1981 & $1.014,5$ & 1974 & $5.100,1$ & 1979 & 85,8 & 22 & 1,41 & 71,0 \\
\hline 2002 & $1.001,4$ & 1981 & $5.041,1$ & 1981 & 83,1 & 23 & 1,35 & 74,2 \\
\hline 1990 & 989,5 & 2002 & $5.002,1$ & 2002 & 80,6 & 24 & 1,29 & 77,4 \\
\hline 1974 & 984,9 & 1990 & $4.775,8$ & 1990 & 79,9 & 25 & 1,24 & 80,6 \\
\hline 1989 & 977,4 & 2001 & $4.674,1$ & 1989 & 78,4 & 26 & 1,19 & 83,9 \\
\hline 2001 & 940,4 & 1999 & $4.582,1$ & 2001 & 71,6 & 27 & 1,15 & 87,1 \\
\hline 1999 & 881,4 & 1989 & $4.395,0$ & 1999 & 70,9 & 28 & 1,11 & 90,3 \\
\hline 1978 & 789,2 & 1978 & $4.087,3$ & 1978 & 62,1 & 29 & 1,07 & 93,5 \\
\hline 1984 & 671,8 & 1984 & $3.350,6$ & 1984 & 56,8 & 30 & 1,03 & 96,8 \\
\hline
\end{tabular}

Nas Figuras 2a e 2b, apresentam-se as probabilidades de ocorrência e os períodos de retorno para os índices $\mathrm{EI}_{30}$ e $\mathrm{KE}>25$, respectivamente. Por meio delas, torna-se possível a obtenção dos índices de erosividade para quaisquer valores de $\mathrm{T}$ e $\operatorname{Pr}$, e vice-versa.

$\mathrm{Na}$ Figura 2a, observa-se que o período de retorno do valor médio de $\mathrm{EI}_{30}$, para o município de Seropédica (5.960,4 MJ mm ha $\mathrm{m}^{-1} \mathrm{ano}^{-1}$ ), é de 1,97 ano, para uma probabilidade de ocorrência de 50,82\%. Análise semelhante pode ser realizada para o índice KE $>25$ (Figura 2b). É esperado, em média, um valor maior ou igual a 99,2 $\mathrm{MJ} \mathrm{ha}^{-1}$ ano $^{-1}$ (valor médio para a região - Tabela 1) uma vez a cada dois anos, ou seja, sua probabilidade de ocorrência é de aproximadamente 50,0\%.

Com base na distribuição dos dados nas Figuras $2 \mathrm{a}$ e $2 \mathrm{~b}$, foram ajustadas as eqs.(7) $\left(\mathrm{R}^{2}=0,88\right)$ e $(8)\left(\mathrm{R}^{2}=0,92\right)$, respectivamente, para $\mathrm{EI}_{30}$ e $\mathrm{KE}>25$, possibilitando, dessa forma, a estimativa dos índices anuais a partir de quaisquer níveis de probabilidade desejados [eqs.(7) e (8)] ou período de retorno, em anos [eqs.(9) e (10)]. 


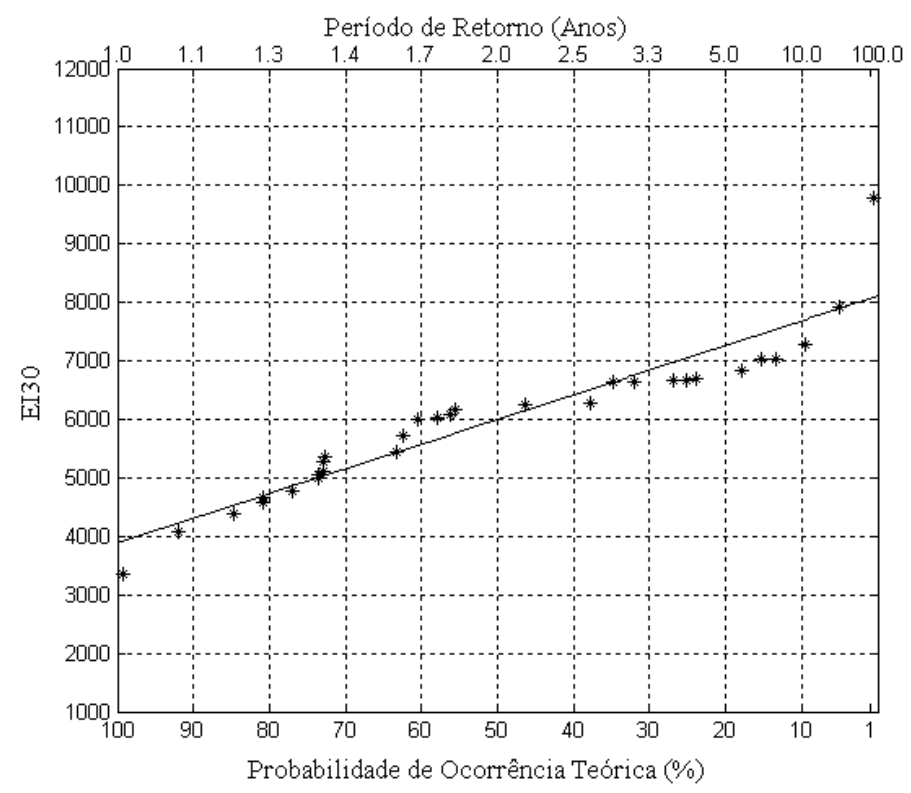

a

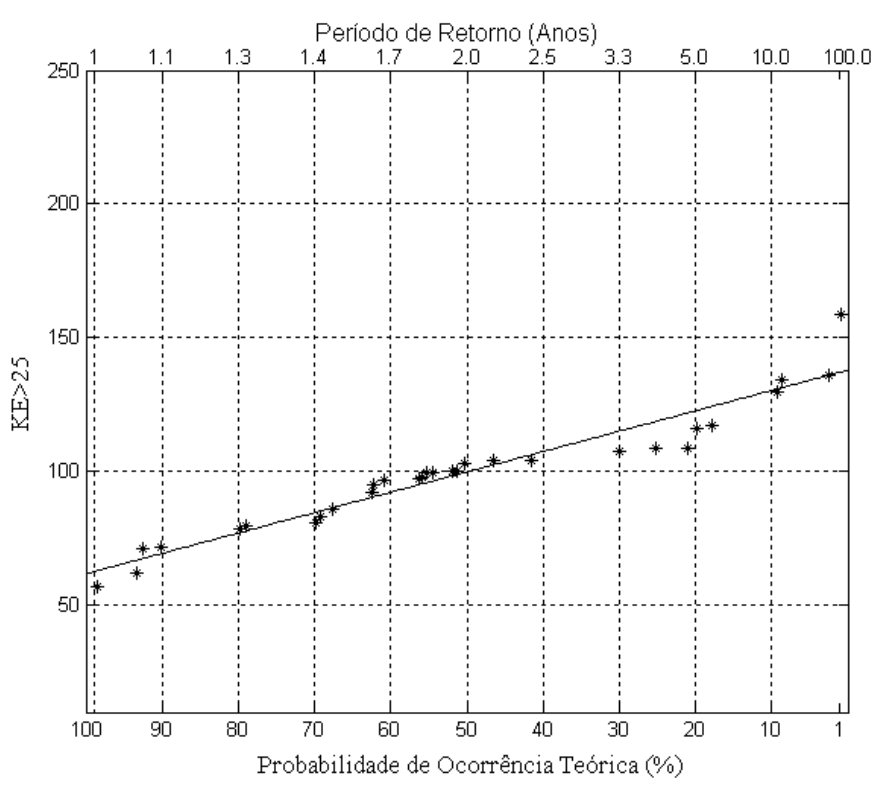

$b$

FIGURA 2. Curva de distribuição da probabilidade de ocorrência e período de retorno do índice de erosividade $\mathrm{EI}_{30}$ (MJ mm ha ${ }^{-1} \mathrm{~h}^{-1} \mathrm{ano}^{-1}$ ) (a) e $\mathrm{KE}>25$ ( $\mathrm{MJ} \mathrm{ha}^{-1} \mathrm{ano}^{-1}$ ) (b), para Seropédica $(\mathrm{RJ})$. Distribution curve of the occurrence probability and return period of erosivity index $\mathrm{EI}_{30}\left(\mathrm{MJ} \mathrm{mm} \mathrm{ha} \mathrm{mb}^{-1} \mathrm{year}^{-1}\right)$ (a) and KE> 25 (MJ ha ${ }^{-1}$ year $^{-1}$ ) (b), for Seropédica (Rio de Janeiro State, Brazil).

$$
\begin{aligned}
& E I_{30}=-42,2353 \operatorname{Pr}+8.106,59 \\
& \mathrm{KE}>25=-0,7612 \operatorname{Pr}+137,88 \\
& \mathrm{EI}_{30}=-4.223,53 \frac{1}{\mathrm{~T}}+8.106,59 \\
& \mathrm{KE}>25=-76,12 \frac{1}{\mathrm{~T}}+137,88
\end{aligned}
$$

De posse das equações, foram calculados os índices de erosividade para a região, esperados para os períodos de retorno de $2 ; 5 ; 10 ; 20 ; 50$ e 100 anos. Na Tabela 3, apresentam-se os valores estimados para Seropédica e também valores de $\mathrm{EI}_{30}$ obtidos por diferentes autores para outras localidades.

TABELA 3. Índice médio anual ( $\overline{\mathrm{IE}})$ e índices anuais de erosividade $\left(\mathrm{EI}_{30}\right)$ para diferentes localidades e períodos de retorno. Medium annual index ( $\overline{\mathrm{IE}}$ ) and annual erosivity index $\left(\mathbf{E I}_{30}\right)$ for different towns and return periods.

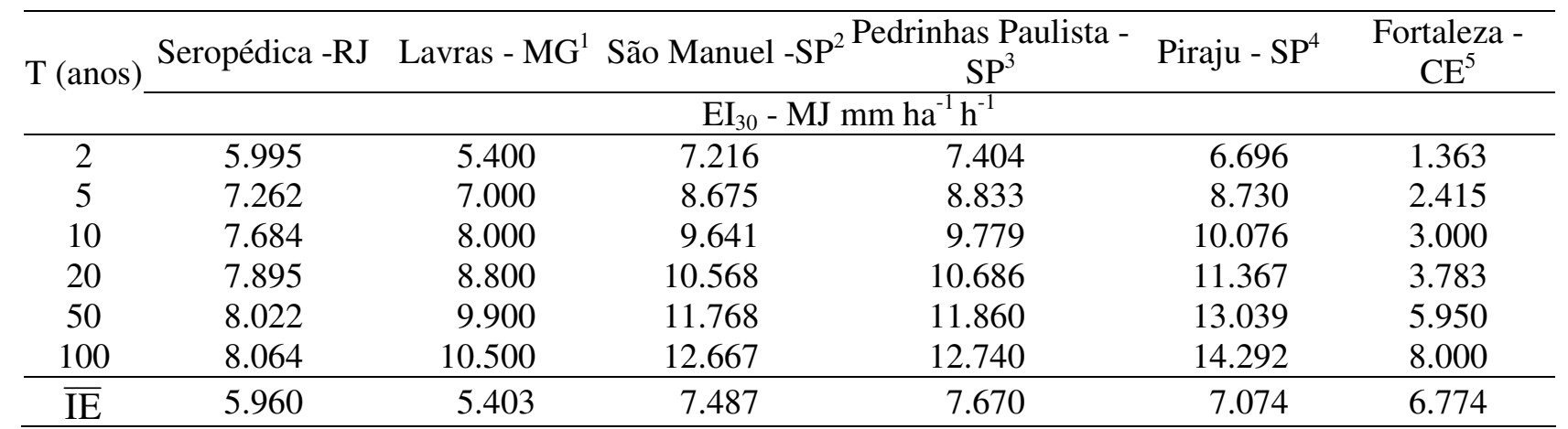

1 - EVANGELISTA et al. (2006); 2 - MORETI et al. (2003a); 3 - MORETI et al. (2003b); 4 - ROQUE et al. (2001); 5 - DIAS \& SILVA (2003) 
É possível verificar que os índices $\mathrm{EI}_{30}$, por estarem associados aos índices pluviométricos, apresentam-se bastante variados para as diferentes localidades. Apesar de MONTEBELLER (2005) ter encontrado $\mathrm{EI}_{30}$ médio anual de até $14.794 \mathrm{MJ} \mathrm{mm} \mathrm{ha}^{-1} \mathrm{~h}^{-1}$ (estação Andorinhas - município de Magé) para o Estado do Rio de Janeiro, o valor de $\mathrm{EI}_{30}$ médio estimado pelo autor foi de 7.184 MJ mm ha ${ }^{-1} \mathrm{~h}^{-1}$, semelhante aos dados médios apresentados na Tabela 3 para algumas localidades no Estado de São Paulo. Analisando ainda a Tabela 3, é possível perceber que Seropédica é a região que apresenta a menor magnitude de variação do $\mathrm{EI}_{30}$ para os períodos de retorno considerados. Isto implica dizer que o índice médio anual de erosividade nessa região apresenta baixa probabilidade de variação ao longo dos anos, principalmente quando comparada a Fortaleza (DIAS \& SILVA, 2003). Com exceção dessa mesma localidade, é possível perceber que, para baixos períodos de retorno (2 e 5 anos), os valores de $\mathrm{EI}_{30}$ apresentam uma mesma tendência para todas as localidades. Em contrapartida, para T igual a 100 anos, o valor médio de $\mathrm{EI}_{30}$ esperado para Fortaleza é o mais próximo daquele observado para Seropédica.

Pelo fato de não terem sido encontrados na literatura trabalhos a respeito de distribuição de probabilidade para o KE $>25$, não foi possível efetuar análise semelhante para este índice. Apesar disso, utilizando a eq.(4), foi possível constatar que, para Seropédica, valores anuais de KE $>25$ da ordem de 99,$8 ; 122,7 ; 130,3 ; 134,1 ; 136,4$ e $137,1 \mathrm{MJ}^{-1}{ }^{-1}$ são esperados, em média, uma vez a cada $2 ; 5 ; 10 ; 20 ; 50$ e 100 anos, respectivamente.

\section{CONCLUSÕES}

A distribuição Log-normal adequou-se satisfatoriamente às séries de índices de erosividade estudadas.

Os índices de erosividade médios anuais estimados para Seropédica apresentaram uma probabilidade de ocorrência de 50,8\%.

Comparativamente a outras localidades, o índice $\mathrm{EI}_{30}$ estimado para Seropédica apresenta baixa magnitude de variação para diferentes períodos de retorno.

\section{REFERÊNCIAS}

BERTOL, I. Avaliação da erosividade da chuva na localidade de Campos Novos (SC) no período de 1981-1990. Pesquisa Agropecuária Brasileira, Brasília, v.29, n.9, p.1.453-1.458, 1994.

BERTONI, J.; LOMBARDI NETO, F. Conservação do solo. São Paulo: Ícone, 1990. 355 p.

CARVALHO, D.F.; MONTEBELLER, C.A.; FRANCO, E.M.; VALCARCEL, R.; BERTOL, I. Padrões de precipitação e índices de erosividade para as chuvas de Seropédica e Nova Friburgo RJ. Revista Brasileira de Engenharia Agrícola e Ambiental, Campina Grande, v.9, n.1, p.7-14, 2005.

CRUZ, F.A. Instalação e calibração de lisímetro de pesagem e determinação da evapotranspiração de referência para a região de Seropédica - RJ. 2005. 56 f. Dissertação (Mestrado em Fitotecnia) Universidade Federal Rural do Rio de Janeiro, Seropédica, 2005.

DIAS, A.S.; SILVA, J.R.C. A erosividade das chuvas em Fortaleza (CE). I - Distribuição, probabilidade de ocorrência e período de retorno - $1^{\underline{a}}$ aproximação. Revista Brasileira de Ciência do Solo, Viçosa - MG, v.27, p.335-345, 2003.

EVANGELISTA, A.W.P.; CARVALHO, L.G.; DANTAS, A.A.A.; BERNARDINO, D.T. Potencial erosivo das chuvas em Lavras-MG: distribuição, probabilidade de ocorrência e período de retorno. Irriga, Botucatu, v.11, n.1, p.1-11, 2006.

FAO. La erosion del solo por el gua-algunas medidas para combetila en las tierras de cultivo. (Caderno Fomento Agropecuário, 81). Roma: Organization de Las Naciones Unidas, 1967. 207 p. 
GONÇALVES, F.A. Erosividade das chuvas no Estado do Rio de Janeiro. 2002. 90 f. Dissertação (Mestrado em Engenharia Agrícola) - Universidade Federal de Viçosa, Viçosa - MG, 2002.

HUDSON, N.W. Soil conservation. Ithaca: Cornell University Press, 1973. 320 p.

LAL, R. Soil erosion on alfisols in western Nigeria. III. Effects of rainfall characteristics. Geoderma, Amsterdam, v.16, p.389-401, 1976.

MAIA NETO, F.F.; CHAVES, I.B. Mapeamento da erosividade das chuvas da Paraíba. In: CONGRESSO BRASILEIRO DE CIÊNCIA DO SOLO, 26., 1997, Rio de Janeiro. Anais... Campinas: Sociedade Brasileira de Ciência do Solo, 1997. 1 CD-ROM.

MATLAB. User's Guide, Natick: The Math Works, 2000.

MONTEBELLER, C.A. Variabilidade espacial do potencial erosivo das chuvas no Estado do Rio de Janeiro. 2005.103 f. Dissertação (Mestrado em Ciência do Solo) - Universidade Federal Rural do Rio de Janeiro, Seropédica, 2005.

MORETI, D.; CARVALHO, M.P.; MANNIGEL, A.R.; MEDEIROS, L.R. Importantes características de chuva para a conservação do solo e da água no município de São Manuel - SP. Revista Brasileira de Ciência do Solo, Viçosa - MG, v.27, n.4, p.713-725, 2003a.

MORETI, D.; MANNIGEL, A.R.; CARVALHO, M.P. Fator erosividade da chuva para o município de Pedrinhas Paulista, Estado de São Paulo. Acta Scientiarum: Agronomy, Maringá, v.25, n.1, p.137-145, 2003b.

ROQUE, C.G.; CARVALHO, M.P.; PRADO, R.M. Fator erosividade da chuva de Piraju - SP: distribuição, probabilidade de ocorrência, período de retorno e correlação com o coeficiente de chuva. Revista Brasileira de Ciência do Solo, Viçosa - MG, v.25, n.1, p.147-156, 2001.

RUFINO, R.L.; BISCAIA, R.C.M.; MERTEN, G.H. Determinação do potencial erosivo da chuva do Estado do Paraná através da pluviometria: terceira aproximação. Revista Brasileira de Ciência do Solo, Viçosa - MG, v.17, p.439-444, 1993.

VAL, L.A.; BAHIA, V.G.; FREIRE, J.C.; DIAS JÚNIOR, M.S. Erosividade das chuvas em LavrasMG. Ciência e Prática, Lavras, v.10, n.2, p.199-209, 1986.

VILLELA, S.M.; MATOS, A. Hidrologia aplicada. São Paulo: McGraw-Hill, 1975. 245 p.

WISCHMEIER, W.H.; SMITH, D.D. Rainfall energy and its relationship to soil loss. Transaction American Geophysical Union, Washington, v.39, p.285-291, 1958. 